\title{
Comprensión lectora y redacción académica en estudiantes de la Facultad de Ingeniería Industrial y de Sistemas en una universidad pública ${ }^{1}$
}

\section{Reading comprehension and academic writing in engineering students from the Faculty of Industrial and Systems Engineering at a public university}

\author{
Roxana Abanto Atauje \\ Universidad Nacional Mayor de San Marcos, Lima, Perú \\ raa.linguistica@gmail.com
}

\begin{abstract}
Resumen
El desarrollo de la investigación tiene como propósito comprobar la relación directa y significativa existente entre la comprensión lectora y la redacción de textos en una universidad de Lima Metropolitana. El enfoque de la investigación es cuantitativo basado en el diseño descriptivo-correlacional. La muestra estuvo conformada por 59 estudiantes de la Facultad de Ingeniería Industrial y de Sistemas, en una universidad de Lima Metropolitana, situada en la ciudad de Lima, Perú. La muestra se determinó gracias un cálculo estadístico; sin embargo, se incrementó a 71 estudiantes durante la aplicación de las pruebas de comprensión lectora y de redacción académica, con el fin de que sea más representativa. Para las técnicas de recolección de datos se aplicó la observación, el análisis documental y el análisis estadístico. Se concluye que existe una relación directa y significativa entre los niveles literal e inferencial y la redacción académica, mientras que el nivel crítico no cumple con dicha relación.
\end{abstract}

Palabras clave: Comprensión lectora, redacción académica, nivel literal, nivel inferencial y nivel crítico.

\begin{abstract}
The purpose of this research is to know the relationship between reading comprehension and text writing at a university in metropolitan Lima. The research approach is quantitative based on the descriptive-correlational design. The sample consisted of 59 students from the Faculty of Industrial and Systems Engineering, at a university of metropolitan Lima, located in the city of Lima, Peru. The sample was obtained thanks to a statistical calculation; however, it was increased to 71 students during the application of the reading comprehension and academic writing tests, in order to make it more representative. For data collection techniques, observation, documentary analysis and statistical analysis were applied. It is concluded that there is a direct and significant relationship between literal and inferential levels and academic writing, while critical level does not comply with that relationship.
\end{abstract}

Keywords: Keywords: Reading comprehension, academic writing, literal level, inferential level and critical level.

1 El presente artículo se basa en la investigación denominada Comprensión lectora y redacción académica en estudiantes universitarios en la Facultad de Ingeniería Industrial y de Sistemas en una universidad de Lima Metropolitana, realizada por la autora.

Recibido: 29.06.19

Aceptado: 15.08 .19 


\section{Roxana Abanto Atauje}

\section{Introducción}

Los estudios acerca de la comprensión lectora y la redacción académica evidencian una clara relación entre ambas variables. Por un lado, una de las preocupaciones de los docentes de Educación Superior es la deficiencia que demuestran los estudiantes en cuanto a la redacción académica, la promoción de prácticas de escritura resulta ser un desafío para los docentes de nivel superior, ya que los estudiantes universitarios deben transformarse en escritores competentes de los géneros académicos correspondientes a su campo profesional y para lo cual es vital la participación del docente (Capomagi, 2013, p. 31).

Por otro lado, la falta de interés que muestra el alumnado hacia la lectura trae como consecuencia las notorias deficiencias en el campo de la redacción académica. La comprensión de textos es también una debilidad que presentan los estudiantes, evidenciada a través de las respuestas obtenidas a las preguntas de los niveles literal, inferencial y crítico de la comprensión lectora, lo cual mostraría un analfabetismo total, esto es, la pobreza de la preparación sistemática o asistemática y el conocimiento previo, que le permita entender el significado de un texto.

Por ello, el presente análisis propone la existencia de una estrecha relación entre los niveles de la comprensión lectora: literal, inferencial y crítico y la redacción de textos académicos, la cual es directa y significativa. Por lo que se toma como muestra a los estudiantes de ingeniería de primer, tercer y quinto año.

\section{Problema de investigación}

La falta de interés que muestra el alumnado hacia la lectura trae como consecuencia las notorias deficiencias en el campo de la redacción académica. La comprensión lectora es también una debilidad que presentan los estudiantes, evidenciada a través de las respuestas obtenidas a las preguntas de los niveles literal, inferencial y crítico de la comprensión lectora, lo cual mostraría un analfabetismo total, esto es, la pobreza de la preparación sistemática o asistemática y el conocimiento previo, que le permita entender el significado de un texto.

Así mismo, aparece en nuestro contexto el fenómeno del analfabetismo funcional, el cual se presenta cuando el estudiante influenciado por su entorno social pierde la habilidad de leer, habilidad que fue adquirida en años anteriores. Esto debido a la contribución del contexto cultural y familiar, que muchas veces suele ser limitado, poco motivador, restrictivo en algunos casos y por el cual se va perdiendo el hábito de la lectura. 
Los estudiantes universitarios suelen pensar que no es importante el saber escribir con propiedad y siguiendo ciertas normas básicas para una buena redacción, por lo que le restan la relevancia que merece la redacción de textos académicos o científicos, situación más notoria en ramas como las ciencias. Una clara muestra de ello es que los docentes universitarios cuando requieren que sus estudiantes argumenten sus respuestas en los exámenes escritos, restan importancia a la corrección de los errores ortográficos o de redacción. Lo mismo sucede en la ponderación de los criterios de evaluación en otros instrumentos de evaluación del aprendizaje utilizados en el ámbito universitario como la monografía, los informes, los ensayos y los resúmenes.

En el ámbito mundial, en España, el Programa Internacional para la Evaluación de Competencias de los Adultos (PIAAC: Program for the International Assessment of Adult Competencies), auspiciado por la Organización para la Cooperación y el Desarrollo Económicos (OCDE), conocido como el PISA para adultos, se encarga de la evaluación del rendimiento en comprensión lectora, comprensión matemática y la resolución de problemas en entornos informáticos entre la población de 16 a 65 años. Este programa reportó que la población española entre 16 y 65 años obtuvo 21 puntos por debajo del promedio de la OCDE y 19 puntos por debajo del promedio de la Unión Europea (UE), por lo que, dentro de la UE, España aparece en la penúltima posición en comprensión lectora, teniendo a Italia, en la última. Mientras que los países con mejores resultados son Japón (296), Finlandia (288) y los Países Bajos (284).

En el siglo xxi, la carrera de Ingeniería es una de las que tiene mayor demanda entre los jóvenes que egresan de las instituciones educativas, siendo importante desarrollar en un profesional Ingeniero algunas competencias específicas durante su formación pre-profesional como el análisis, procesamiento de información, la edición y redacción de informes. Esto, debido a que la sociedad actual demanda el manejo de las habilidades cognitivas que se desarrollan a través de la lectura y la escritura, y un futuro Ingeniero debe comunicarse eficazmente, ya sea de forma oral o escrita.

En relación con Latinoamérica, Natale y Stagnaro (2012) afirman que es importante fortalecer las habilidades de lectura y de comunicación oral y escrita en el proceso de formación de los estudiantes de Ingeniería, para mejorar el desempeño del ingeniero en el ámbito profesional, ya que, en el campo laboral, el ingeniero debe interactuar no sólo con personal de rangos jerárquicos superiores, sino también con rangos inferiores como son los operarios, los proveedores y/o los clientes. 
En el Perú ${ }^{2}$ luego de una búsqueda en bases de datos online se evidenció que no existen estudios relacionados con la comprensión lectora y la redacción académicas en estudiantes universitarios, y menos aún en la especialidad de Ingeniería. Sin embargo, se observan datos estadísticos acerca del rendimiento en comprensión lectora provenientes de la prueba PISA (2015), aplicada por la Organización para la Cooperación y el Desarrollo Económicos (OCDE). EL Perú ocupa el puesto 64, con un promedio de 398 puntos, respecto de los 493 que se establece como calificación mínima, es decir que aún existe una brecha estudiantil en relación con otros países de América Latina (OCDE, 2016).

Esta situación latente preocupa tanto a los docentes como a la familia, ya que refleja un rendimiento muy bajo en procesos de oralidad, escucha, análisis, decodificación, construcción de significados, entre otros, que el estudiante debe llegar a dominar para llegar a niveles avanzados de educación. Esto luego es evidente en el ámbito de la Educación Superior universitaria, donde se observa que los alumnos tienen dificultades serias para comprender un texto y, por ende, para redactar.

Lo mencionado líneas arriba va en contraposición de lo expresado por Narvaja (2006, citado por Olave-Arias, Rojas-García, \& Cisneros-Estupiñán, 2013, p. 461), cuando sostiene que [en la universidad] un alumno lea e interprete la dimensión polémica de los discursos, establezca relaciones entre texto-autor, texto-texto y texto-conocimientos previos; esto en concordancia con la finalidad de lectura en la universidad, donde se orienta hacia una dimensión de análisis que requiere una cierta experiencia de lectura, mientras que en la escuela se pretende formar hábitos lectores, apoyar constructos teóricos y desarrollar comprensión lectora. (Olave-Arias et al., 2013, p. 461).

Según el estudio realizado por el Instituto de Opinión Pública (IOP) de la Pontificia Universidad Católica del Perú (PUCP), en setiembre de 2015, el 17.8\% de los jóvenes de entre 18 y 29 años lee cotidianamente y solo el $18.5 \%$ de entrevistados indicó que había asistido a una biblioteca en los últimos 12 meses. Es decir, más del $80 \%$ de peruanos no presenta un acercamiento hacia la lectura de textos (IOP, 2015). Esto se vincula de forma directa con la capacidad de redactar un texto académico por parte de un estudiante universitario.

2 Pese a que son escasos los estudios acerca de la relación entre la comprensión lectora y la redacción académica, se cuenta con datos estadísticos que son de utilidad para la presente investigación. 
Por lo expuesto, se propone la existencia de una estrecha relación entre la comprensión lectora y la redacción de textos académicos, esto además apoyado por Espinosa (1998, p. 57) quien sostiene que existe una indivisible dialéctica entre dos actividades fundamentales practicadas con la lengua: leer, escribir, releer, revisar, reescribir, leer nuevamente. Así mismo, afirma que este aprendizaje es continuo, incidental e inconsciente, y que se aprende desde la escuela con la ejercitación.

En ese sentido, el problema de la comprensión lectora y la redacción de textos académicos se hace más evidente en el aula de clases, donde los estudiantes universitarios evidencian sus deficiencias en cuanto a la escritura y, en la mayoría de los casos, al momento de presentar avances escritos de sus estudios, prefieren tomar textos de internet de manera literal, con el fin de disminuir tiempos, completar tareas y automatizarse en la redacción. Todo ello, generará, retrasos y defectos en su comprensión de lectura.

Las investigaciones mostradas dan cuenta de que existen estudios acerca de la comprensión lectora y la redacción de forma independiente, por lo que se destaca el hecho de que se realicen estudios de manera conjunta, que demuestren la relación de ambas variables.

\section{Elementos teóricos}

\subsection{Comprensión lectora}

Es el «[...] proceso que se construye y desarrolla gracias a los aportes del texto y por el conocimiento del lector previamente, es decir, el oyente o lector participa activamente en la construcción o reconstrucción del significado intencionado por el hablante/autor, poniendo en juego una serie de procesos mentales [...]» (Peronard, Parodi \& Núñez, 1997; citado por Cáceres, Donoso \& Guzmán, 2012, p. 60).

La comprensión lectora es un proceso cognitivo que consta de tres niveles, el literal, el inferencial y el crítico.

\subsubsection{Nivel literal}

El nivel literal se encuentra explícito en el texto, es todo aquello que está escrito, detalles como nombres, personajes, lugares, fechas, sucesos o hechos que están plasmados en la obra, así como las ideas principales y secundarias, entre otros. 
Para poder identificar estos detalles se realiza una lectura rápida, por lo cual se le puede considerar como un nivel de comprensión básico.

Este es el primer nivel de lectura, el cual se limita a extraer la información que se tiene en el texto, sin agregarle ningún valor interpretativo. Los procesos que involucran este nivel son los siguientes: la observación, la comparación y la relación, la clasificación jerárquica, el análisis, la síntesis y la evaluación. (Pérez, 2006).

Según Strang (1965), Jenkinson (1976) y Smith (1989), citados por Gordillo y Flórez (2009) en la Revista Actualidades Pedagógicas, el reconocimiento consiste en la localización e identificación de los elementos del texto, que pueden ser:

a. de ideas principales: la idea más importante de un párrafo o del relato;

b. de secuencias: identifica el orden de las acciones;

c. por comparación: identifica caracteres, tiempos y lugares explícitos;

d. de causa o efecto: identifica razones explícitas de ciertos sucesos o acciones (p. 97).

Los mencionados autores sostienen que existen dos subniveles en el nivel literal: el primero es la lectura en un nivel primario, conocido también como el nivel 1 , en el que se pretende reconocer aquellos sucesos que están expresados en el texto, además de identificar algunos elementos como lugares, fechas, personajes, en caso lo hubiera, acciones, entre otros.

El segundo subnivel es la lectura literal en profundidad, al cual también se le denomina nivel 2, en el que se realiza una lectura más minuciosa en la cual se puede determinar la macroestructura textual, que es la parte más general y global, esto es el tema o asunto del texto.

\subsubsection{Nivel inferencial}

Este es el segundo nivel de lectura, en el cual se realizan inferencias acerca de lo leído. La inferencia «se interpreta a partir de enunciados textuales generales o particulares de modo que el lector lo único que hace es sacar a la luz los sobreentendidos» (Pérez, 2006, p. 78).

En este nivel se requiere que el lector realice deducciones a partir de las ideas expresadas en el texto, debe decodificar lo que el autor trata de expresar, es decir interpretar los signos a partir de su experiencia previa, y tratar de definir el significado del mensaje o información del emisor. Los conocimientos previos le ayudarán al lector a elaborar predicciones, además de realizar valiosas aportaciones a partir de sus saberes preliminares. 
En concordancia, Pérez (2006) considera lo siguiente: «El proceso de inferencia introduce al lector en un mundo de interpretaciones que va más allá de lo explícito. Permite el uso del pensamiento crítico, emitir juicios valorativos e interpretar las ideas a la luz de las experiencias previas». (p. 77). Es decir que a partir de realizar una buena interpretación del texto o de lo que este pretende transmitir, el lector puede elaborar un juicio de valor en relación con el tema que plantea el autor. Realizar inferencias de un texto implica descifrar aquello que no está escrito en él considerando las ideas que se manifiestan expresamente en la lectura.

Para concretar este segundo nivel de comprensión lectora, se realizan diversos procesos cognitivos, adicionalmente a los mencionados en el primer nivel, el literal. Por ello, Strang (1965), Jenkinson (1976) y Smith (1989), citados por Gordillo y Flórez (2009) en la Revista Actualidades Pedagógicas:

Este nivel puede incluir las siguientes operaciones:

a. inferir detalles adicionales que, según las conjeturas del lector, pudieron haberse incluido en el texto para hacerlo más informativo, interesante y convincente;

b. inferir ideas principales, no incluidas explícitamente;

c. inferir secuencias sobre acciones que pudieron haber ocurrido si el texto hubiera terminado de otra manera;

d. inferir relaciones de causa y efecto, realizando hipótesis sobre las motivaciones o caracteres y sus relaciones en el tiempo y el lugar. Se pueden hacer conjeturas sobre las causas que indujeron al autor a incluir ciertas ideas, palabras, caracterizaciones, acciones;

e. predecir acontecimientos sobre la base de una lectura inconclusa, deliberadamente o no;

f. interpretar un lenguaje figurativo, para inferir la significación literal de un texto (p. 98).

En general, los autores sostienen que, en algunos casos, el texto se centra en un tema en específico, pero las proposiciones que se observan no siempre explicitan los puntos principales de que trata la lectura. Justamente, en este tipo de textos, el estudiante debe utilizar la capacidad de inferir cuáles son las ideas principales a partir de la comprensión del significado de la lectura.

Así mismo, el docente puede realizar algunas preguntas que le permitan al estudiante deducir qué otro final pudo haber tenido el texto o historia que plantea el autor. Además, el lector podrá realizar algunas hipótesis acerca de las causas y efectos acerca de determinados hechos que ocurren en la lectura. 


\section{Roxana Abanto Atauje}

\subsubsection{Nivel crítico}

Este es el tercer nivel, el cual requiere de los procesos mencionados en los dos anteriores niveles, además establece relaciones analógicas de diferente índole y emite juicios de valor acerca de los leído (Pérez, 2006, p. 77).

En este nivel de comprensión lectora, el lector expresa su valoración respecto del tema planteado en el texto, ya que el nivel crítico tiene un carácter evaluativo. Se consideran los saberes previos del lector y la comprensión profunda del sentido del texto y de lo que realmente transmite o desea manifestar el autor.

La valoración de un texto solo se puede hacer luego de concluir con la lectura y de la comprensión de esta. Cuando se llega a este nivel se logra la lectura eficiente, puesto que se llega más allá de lo explícito e implícito en el texto. El lector muestra una actitud independiente en relación con la postura del autor. «La lectura crítica tiene un carácter evaluativo, en el que interviene la formación del lector, su criterio y conocimientos de lo leído» (Gordillo \& Flórez, 2009, p. 98).

La importancia de realizar una lectura crítica es que esto le permitirá al estudiante, en primer lugar, investigar acerca de un tema en específico que plantea el texto, con lo cual alcanzará el conocimiento requerido para la comprensión lectora y la posterior valoración. En segundo lugar, estas actividades habituarán al estudiante a mejorar en cuanto a las estrategias que utiliza para leer $y$, por ende, en su formación como lector.

\subsection{Producción de textos escritos}

La redacción es una actividad que amerita un gran esfuerzo cognitivo por parte del autor, en este caso, los estudiantes universitarios, investigadores, docentes, quienes expresan sus conocimientos acerca de un determinado tema, además les permite profundizar más en cuanto a la información que debe manejar para la redacción del texto y, por ende, los conocimientos con los que contaban se transforman y aprenden más, ya que deben revisar diversas fuentes confiables y académicas.

Smith (1978), citado por Bono y De la Barrera (1996, p. 4), señala que los mitos de la escritura nos revelan que la capacidad de escribir no es privativa de unos pocos elegidos, los llamados escritores (de reconocida trayectoria), sino que nos pertenece a todos los que estemos dispuestos a desarrollarla. Las ideas surgen de la misma escritura, es a través del propio acto de escribir como creamos y exploramos nuevas ideas. 
Comprensión lectora y redacción académica en estudiantes...

La producción de textos académicos ${ }^{3}$ es un proceso complejo y sistemático, que requiere de un trabajo intelectual arduo, este tipo de textos se suelen elaborar en las universidades por investigadores, docentes, estudiantes universitarios de diferentes disciplinas. La elaboración de dichos textos se sostiene de algunas fases, la planificación, la textualización, la revisión.

El acceso a los textos escritos necesita una actividad cognitiva que, en el caso de la escritura, implica la preparación y la composición de borradores sucesivos y la revisión, la reescritura y la transformación del texto a medida que se escribe. El conocimiento del proceso de producción escrita favorece la intervención docente para el logro de mejores producciones en sus alumnos (Capomagi, 2013, p. 31).

Además de tomar en cuenta el fondo, como la generación de ideas, planificación, la escritura como tal, textualización, la evaluación o corrección del texto, revisión, también se debe considerar el aspecto formal, en este plano se observa la gramática: la morfología, la sintaxis, la organización y la combinación de las oraciones o ideas, la semántica; adicionalmente, al redactar es importante cómo se expresan las ideas, los signos de puntuación que se utilizan, puesto que la ubicación de la coma, por ejemplo, puede variar el sentido de lo que se desea manifestar en un escrito.

[...] quien escribe debe atender a aspectos de ortografía, de uso del léxico, de arreglo sintáctico, de comunicación de significados, de estilo y organización textual, y, sobre ellos, orquestar y coordinar una producción que tiene mucho de creativa y original. (Hayes y Flower, 1987; Cooper y Matsuhashi, 1983; Martlew, 1983; Scardamalia y Bereiter, 1985, citados por Díaz \& Hernández, 2002, p. 310).

Los textos están compuestos por ideas principales y secundarias, las cuales se interrelacionan entre sí gracias a los conectores lógicos, a los signos de puntuación y, en general, a la organización del texto. Cuando las ideas que redacta el autor tienen una secuencia lógica y existe armonía y/o relación entre todos los párrafos, se considera que es un texto que cumple con dos características principales: coherencia y cohesión.

Del mismo modo, los textos que se producen en un contexto académico deben cumplir con cierto rigor o, dicho de otro modo, algunas características básicas:

3 Son aquellos que se realizan en el ámbito universitario, estos cumplen con un conjunto de requisitos, existen diferentes géneros discursivos en el contexto universitario. 


\section{Roxana Abanto Atauje}

[...] le exige al escritor ser preciso, sistemático y ordenado en la exposición de las ideas; le demanda que seleccione con mayor rigor los significados y las ideas en relación con las intenciones comunicativas que persigue [...]. (Díaz \& Hernández, 2002, p. 311)

En suma, es importante mantener un orden para redactar un texto académico, para una mejor cohesión de todos los párrafos, lo cual logrará que haya una mejor presentación de las ideas u oraciones planteadas en el texto y, a su vez, se facilitará el propósito de la lectura: la trasmisión de un mensaje.

\subsection{Etapas de la producción de textos escritos}

Fayol (1991; citado por Díaz y Hernández, 2002, p. 311) plantea dos dimensiones: una funcional y otra estructural. En cuanto al aspecto funcional, se considera el qué decir, cómo decirlo, para quién decirlo, para qué y por qué decirlo. En relación con el aspecto estructural, la producción de textos escritos consta de tres etapas: planificación, textualización o generación del escrito y revisión. A continuación, se desarrollarán estos tres subprocesos:

\subsubsection{Planificación}

En esta etapa se requiere que el estudiante realice un plan de redacción acerca del tema que tratará en el texto académico. El autor debe partir de algunos puntos importantes: el tema que desarrollará, el tipo de lenguaje que utilizará, lo cual dependerá de a quiénes va dirigido el texto, entre otros aspectos a los cuales aluden Díaz y Hernández (2002) en la siguiente cita:

[...] se genera una representación abstracta (en la mente del escrito) de aquello que desea escribir [...], en función de las cuatro preguntas funcionales [...] (sobre los aspectos temáticos, comunicativos, lingüístico-organizativos, instrumentales, etcétera) y su compleja interacción entre sí [...] se denomina "plan de escritura», que en esencia es un plan jerarquizado de metas y submetas sobre cómo operará el proceso compositivo en forma global y sobre la naturaleza del producto escrito que se intentará lograr.

(p. 312).

En esa línea, Cassany (2003) sostiene que es importante la planificación para redactar un texto, es decir el generar un esquema mental de lo que se desea plasmar en el texto, en esta etapa también se debe considerar lo que se desea lograr con el texto, la intención que se tiene en relación con el lector. «[...] esquema mental 
del texto que van a escribir, se formulan una imagen de lo que quieren escribir, y también de cómo van a trabajar. Se marcan objetivos» (p. 262).

En el texto se evidencia la planificación cuando este cuenta con una secuencia de oraciones que en su conjunto forman parte de párrafos, que deben cumplir con un rasgo importante: la cohesión, para lo cual se debe contar con el apoyo de conectores lógicos y el buen uso de los signos de puntuación. Los párrafos que forman parte del texto se denominan macroposiciones. A continuación, se describe en qué consisten las macroposiciones:

Una macroestructura está formada por un conjunto de macroproposiciones, es decir, proposiciones que sintetizan o cubren varias proposiciones, que reflejan los temas o asuntos más generales e importantes del discurso. (Coherencia global) y que nos dan las ideas centrales del mismo.

(Ochoa \& Gómez, 2011, p. 7)

Cuando el texto está bien cohesionado, entonces se considera que cumple con otro requisito importante: la coherencia, cuando todas las partes del texto desarrollan un tema específico y todas las ideas, principales y secundarias, guardan relación entre sí.

\subsubsection{Textualización}

Esta fase consiste en el proceso de elaboración del texto, en el cual se unen las palabras para formar unidades mayores, oraciones, y el conjunto de estas que forman párrafos. Estas deben guardan relación entre sí, además deben cumplir con una serie de normas ortográficas y gramaticales. A continuación, Díaz y Hernández (2002) explican brevemente lo siguiente acerca de la textualización:

[...] ocurre la realización del plan elaborado y la producción formal de frases coherentes y con sentido. Durante toda la textualización tiene lugar una serie de operaciones relacionadas con la traducción de paquetes semánticos (explicaciones, proposiciones, códigos visuales, etcétera) almacenados en la memoria a largo plazo en información lingüística, tomando una serie de decisiones recurrentes sobre reglas de correspondencia grafema-fonema, ortografía, puntuación, reglas gramaticales, sintaxis, procesos semánticos y textuales.

(p. 312)

La textualización debe cumplir con tres características: coherencia, cohesión, adecuación. La coherencia consiste en la relación que guardan todos los párrafos de tal manera que conforman una unidad. Es decir, no deben existir contradicciones 
entre los enunciados, La cohesión se trata de la unión que existe entre párrafo y párrafo en el texto, con el apoyo de los conectores lógicos, los signos de puntuación y los mecanismos de cohesión. Finalmente, la adecuación, propiedad por la que el texto cumple con ciertas normas sociales, personales, lingüísticas.

Es esencial que en la textualización se logre establecer una correspondencia adecuada entre el esquema de planificación subjetivo y el arreglo secuencial lingüístico, cuidando aspectos de cohesividad, coherencia, adecuación e inteligibilidad (Díaz \& Hernández, 2002, p. 312). En suma, la redacción de un texto académico implica un proceso en el que se utilizan recursos lingüísticos, sociales y personales, con la finalidad de que el producto pueda ser comprendido atractivo para el lector.

\subsubsection{Revisión}

La fase de la revisión implica la elaboración de un primer borrador en el cual se pueden observar los aciertos y desaciertos, los errores se deben subsanar posteriormente para lograr un texto «limpio» o perfeccionado.

Díaz y Hernández (2002) sostienen lo siguiente:

[...] consiste en mejorar o refinar los avances y ejemplares (que podríamos llamar «borradores» logrados en la textualización. En este subproceso, se incluyen las actividades de lectura de lo escrito, actividades de diagnóstico y evaluación correctiva, regulados esencialmente para valorar el grado de satisfacción del plan inicial) (p.315).

Según Bereiter y Scardamalia (1987; citados por Díaz \& Hernández, 2002) sostienen que existen tres operaciones básicas: comparación, por la cual se establece una relación entre la representación del texto planificado y la representación que en ese momento se está elaborando, y no siempre habrá diferencias sensibles entre ellas; en la operación de diagnóstico se intenta establecer una valoración del porqué del ajuste existente cuando este sea el caso (... e incluso se puede valorar la posibilidad de cambiar el plan); y por último, en la operación de corrección, se decide seguir una posible alternativa que corrija el problema detectado y, luego, proceder a textualizarla (p. 315).

Es importante que, al realizar la corrección del texto o primer borrador, el autor no se olvide la intención que el autor tuvo desde el inicio, es decir, no se debe olvidar el sentido de lo que se deseaba transmitir al lector. Las correcciones que se realicen deben estar enfocadas en los aspectos gramatical, si alguna oración no 
tiene una sintaxis aceptable, ortográfico, si alguna palabra no está correctamente escrita, ortotipográfico.

Del mismo modo, la tipografía del texto, que debe buscar la claridad y legibilidad de este, y de estilo, que muestra el sello del autor. Al respecto, Cassany (2003) afirma que mientras escribe y relee el texto, el autor lo revisa e introduce modificaciones y mejoras. Estos cambios afectan sobre todo al contenido del texto: al significado (p. 262).

\section{Método}

El presente artículo de investigación es cuantitativo, empírico, ya que el objeto de estudio es observable en la realidad. Es correlacional, ya que mide el grado de relación entre las variables, transversal, puesto que se trabaja con grupos ya formados.

\subsection{Población}

La población se conforma por los estudiantes ${ }^{4}$ de la Facultad de Ingeniería Industrial y de Sistemas de una universidad pública de Lima Metropolitana pertenecientes al semestre 2018 II, los cuales se seleccionaron para conocer el nivel de comprensión lectora y redacción académica con la que inician dichos estudiantes de Ingeniería de primer año y cuál será el proceso de evolución con el transcurso del tiempo en la vida universitaria, por lo cual también se toma en cuenta a los alumnos de tercer y quinto año.

Por el tamaño poblacional mostrado fue necesario obtener una muestra aleatoria para estudios de correlación. Se utilizó la siguiente fórmula:

$$
n=\left(\frac{z_{1-\alpha / 2}+z_{1-\beta}}{\frac{1}{2} \ln \left(\frac{1+r}{1-r}\right)}\right)^{2}+3
$$

Donde:

- $\mathrm{r}=$ correlación que se desea detectar

- $\ln =$ logaritmo neperiano.

- $\mathrm{Z}_{1}-\beta=$ Coeficiente de distribución norma de poder.

- $\mathrm{Z}_{1}-\alpha / 2=$ Coeficiente de distribución norma de seguridad.

4 Para el Muestreo Probabilístico, se tomó en cuenta la cantidad total de estudiantes de primer, tercer y quinto año académico, luego, se aplicó la fórmula estadística. 
A partir de ello se calcula una muestra tomando en cuenta lo siguientes datos: $r=0.3$ (asumido); $Z_{1}-\alpha / 2=1.96 ; Z_{1}-\beta=0.84$. Por lo que la muestra se constituye por 59 estudiantes. Tomando en cuenta el muestreo estratificado se tiene una muestra de 59 estudiantes, que se distribuyó de la siguiente forma, en función de la población elegida de $1 .{ }^{\mathrm{er}}, 3 \cdot{ }^{\mathrm{er}}$ y $5 \cdot{ }^{\mathrm{to}}$ año de estudios.

Tabla 1. Distribución de estudiantes por año

\begin{tabular}{l|l|l}
\hline Año de estudios & Población & Muestra \\
\hline $1 .^{\text {er }}$ & 348 & 18 \\
\hline $3 .^{\text {er }}$ & 307 & 16 \\
\hline $5 .^{\text {to }}$ & 469 & 25 \\
\hline Total & 1124 & 59 \\
\hline
\end{tabular}

Fuente: Tomado de la Oficina de Servicios Académicos de una Facultad de Ingeniería Industrial y de Sistemas, solo los datos de la población.

\section{Resultados}

La presentación de los resultados se realizará a partir del análisis estadístico (cuantitativo) con la finalidad de demostrar la relación entre las variables comprensión lectora y redacción académica. Para ello, se muestran algunas tablas en las cuales se aplicó el coeficiente de correlación de Spearman (rho), que es una prueba de correlación, de asociación o de interdependencia entre dos variables aleatorias, que pueden ser continuas o discretas (en este caso, discretas).

\subsection{Relación entre la comprensión lectora y la redacción académica}

Tabla 2. Prueba de correlación de Spearman

\begin{tabular}{l|l|l|l}
\cline { 3 - 3 } $\begin{array}{l}\text { Rho de } \\
\text { Spearman }\end{array}$ & $\begin{array}{l}\text { Puntaje de } \\
\text { RA }\end{array}$ & $\begin{array}{l}\text { Coeficiente } \\
\text { de correlación }\end{array}$ &, $324^{* *}$ \\
\hline & Sig. (bilateral) &, 006 \\
\hline N & 70 \\
\hline
\end{tabular}

CL: Comprensión Lectora; RA: Redacción Académica. 
Comprensión lectora y redacción académica en estudiantes...

\section{Interpretación:}

El valor de $\mathrm{p}$ hallado es menor que $0.05(\mathrm{p}=0,006)$, por lo que existe evidencia para rechazar la hipótesis nula, es decir, que se descarta que el coeficiente de correlación sea o. Por lo tanto, y observando el coeficiente de correlación Rho Spearman positivo, se demuestra que existe relación directamente proporcional entre la compresión lectora y la redacción académica $(r=0.324)$, teniendo un coeficiente de correlación bajo. Todo ello nos lleva decir que a mayor comprensión lectora mayor redacción académica y a menor comprensión lectora menor redacción académica. Es cierto que, según el valor hallado, se considera una correlación débil, pero a la vez significativa, lo cual para las ciencias de la educación es relevante.

\subsection{Relación entre el nivel literal y la redacción académica}

Tabla 3. Prueba de correlación de Spearman.

\begin{tabular}{l|l|l}
\multicolumn{2}{l}{} & Puntaje total de RA \\
\hline $\begin{array}{l}\text { Nivel_literal } \\
\text { Rho de } \\
\text { Spearman }\end{array}$ & $\begin{array}{l}\text { Coeficiente de } \\
\text { correlación }\end{array}$ &, $286^{*}$ \\
\cline { 2 - 3 } & Sig. (bilateral) &, 016 \\
\hline N & 71 \\
\hline
\end{tabular}

RA: Redacción Académica.

\section{Interpretación:}

El valor de p hallado es menor que 0.05 ( $\mathrm{p}=0,016)$, por lo que existe evidencia para rechazar la hipótesis nula, es decir, que se descarta que el coeficiente de correlación a nivel poblacional sea o. Por lo tanto, y observando el coeficiente de correlación Rho Spearman positivo, se demuestra que existe relación directamente proporcional entre el nivel literal de la compresión lectora y la redacción académica $(r=0.286)$, teniendo un coeficiente de correlación bajo. Todo ello nos lleva decir que a mayor nivel literal de comprensión lectora mayor redacción académica y a menor nivel literal de comprensión lectora menor redacción académica. Es cierto, que, según el valor hallado, se considera una correlación débil, pero a la vez significativa, lo cual para las ciencias de la educación es relevante. 


\subsection{Relación entre el nivel inferencial y la redacción académica}

Tabla 4. Prueba de correlación de Spearman

\begin{tabular}{|l|l|l|l|}
$\begin{array}{l}\text { Rho de } \\
\text { Spearman }\end{array}$ & $\begin{array}{l}\text { Nivel } \\
\text { inferencial }\end{array}$ & $\begin{array}{l}\text { Coeficiente } \\
\text { de correlación }\end{array}$ &, $374^{* *}$ \\
\hline & Sig. (bilateral) &, 001 \\
\hline & N & 71 \\
\hline
\end{tabular}

RA: Redacción Académica.

\section{Interpretación:}

El valor de $\mathrm{p}$ hallado es menor que 0.05 ( $\mathrm{p}=0,001)$, por lo que existe evidencia para rechazar la hipótesis nula, es decir, que se descarta que el coeficiente de correlación a nivel poblacional sea o. Por lo tanto, y observando el coeficiente de correlación Rho Spearman positivo, se demuestra que existe relación directamente proporcional entre el nivel INFERENCIAL de la compresión lectora y la redacción académica $(r=0.374)$, teniendo un coeficiente de correlación bajo. Todo ello nos lleva decir que a mayor nivel literal de comprensión lectora mayor redacción académica y a menor nivel literal de comprensión lectora menor redacción académica.

Es cierto que, según el valor hallado, se considera una correlación débil, pero a la vez significativa, lo cual para las ciencias de la educación es relevante.

\subsection{Relación entre el nivel crítico y la redacción académica}

Tabla 5. Prueba de correlación de Spearman

\begin{tabular}{|c|c|c|c|}
\hline & & & Puntaje total de RA \\
\hline \multirow[t]{3}{*}{$\begin{array}{l}\text { Rho de } \\
\text { Spearman }\end{array}$} & \multirow[t]{3}{*}{ Nivel crítico } & $\begin{array}{l}\text { Coeficiente } \\
\text { de correlación }\end{array}$ &, 052 \\
\hline & & Sig. (bilateral) &, 668 \\
\hline & & $\mathbf{N}$ & 71 \\
\hline
\end{tabular}

RA: Redacción Académica.

24 Lengua \& Sociedad, vol. 18, n. ${ }^{\circ}$ 1, Lima, enero-junio de 2019 


\section{Comprensión lectora y redacción académica en estudiantes...}

\section{Interpretación:}

El valor de p hallado es MAYOR que 0.05 ( $\mathrm{p}=0,668)$, por lo que no existe evidencia para rechazar la hipótesis nula, es decir, que no se descarta que el coeficiente de correlación a nivel poblacional sea o. Por lo tanto, se demuestra que no existe relación entre el nivel cRÍTico de la compresión lectora y la redacción académica.

\section{Discusión}

Acerca de la relación entre la comprensión lectora y la redacción académica, sí existe una relación significativa entre la comprensión lectora y la redacción académica, debido a que, si el estudiante tiene diferentes experiencias en relación con la lectura y si además realiza una lectura óptima, almacenará información y tendrá conocimientos acerca de diversos temas, para luego plasmarlo en un texto.

Tal como señalan Arrieta de Meza y Meza (2006), uno de los factores que demuestran que existe una clara relación entre la comprensión lectora y la redacción académica es la falta de información que suelen tener los estudiantes universitarios para realizar una buena redacción, que se debe al poco interés por la lectura.

Boudghene y Bettadj (2014) sostienen que el docente debe sugerirles a los estudiantes que realicen lecturas variadas de acuerdo con los intereses o preferencias. La intención es que los alumnos puedan anotar aquellas frases que sean significativas. Además, que revisen con frecuencia el periódico local y escribir un resumen acerca de la noticia más importante; todo esto servirá para que luego puedan redactar mejor.

A continuación, se presentarán las relaciones existentes entre los niveles literal e inferencial y la redacción académica, pese a no haber fuentes que evidencien esta relación, se propone lo siguiente:

De acuerdo con Strang (1965), Jenkinson (1976) y Smith (1989), citados por Gordillo y Flórez (2009), el nivel literal reconoce la idea más importante de un párrafo o relato, identifica el orden de las acciones en el texto y reconoce la idea principal del texto.

Sánchez (2003) manifiesta que la escritura es un proceso que consiste en siete etapas, el primer paso de la pre-redacción, que se basa en la selección y límites del tema, es decir acerca de qué se va a escribir, se debe precisar el tema, también se generan algunas ideas, identificar palabras clave o vocablos con una gran carga informativa. Además, se realiza la taxonomía de dichas ideas, en principales, y sus 


\section{Roxana Abanto Atauje}

subdivisiones, secundarias, e incluso las que se considerarán en la introducción y en la conclusión del texto académico.

A partir de lo expuesto, se puede colegir lo siguiente: el estudiante que es capaz de comprender a nivel literal un texto, también sabrá cómo plasmar el tema principal del texto, las macroposiciones, las microposiciones, es decir, podrá generar la estructura semántica del texto o macroestructura.

En cuanto al nivel inferencial, en primer lugar, el lector debe descifrar o interpretar el mensaje del texto con el apoyo de las palabras clave y/o la combinación de estas, las cuales se encuentran en la lectura propuesta. (Pineda \& Lemus, 200o).

Con respecto a la redacción de textos académicos, Sánchez (2003) afirma que, en la fase de escritura del segundo borrador, el estudiante, por lo general, debe reformular lo que planteó al inicio en el texto, es decir debe replantear los párrafos, las ideas propuestas en la introducción, seleccionar mejor lo que colocará en las conclusiones y, de ser necesario, omitir información.

Por ende, se sostiene que para producir un texto académico es importante que el estudiante sea competente en relación con el nivel inferencial de la comprensión lectora, esto es que sea capaz de elaborar inferencias, ya que de esta manera demuestra su conocimiento acerca de diferentes aspectos de un tema y, en general, del mundo. Así mismo, el estudiante será capaz de discernir la información que debe considerar en el texto en el proceso de la redacción académica (especialmente, en la textualización). En caso de que alguna de las proposiciones no sea pertinente con el tema, el estudiante podrá detectarlo con más facilidad y, posteriormente, modificarla en el texto.

Acerca del nivel crítico, el lector manifiesta sus juicios y valoraciones, gracias al conocimiento o saberes previos de diferentes procedencias, además, se da a conocer la ideología del lector. Manifiesta su punto de vista acerca de la lectura con relación a otros textos y se pueden agregar citaciones, alusiones e imitaciones (Perilla, Rincón, Gil \& Salas, 2004).

En el caso de los resultados en cuanto al nivel crítico de la comprensión lectora, la razón principal es que se consideró que el estudiante pudiera seleccionar una alternativa de manera libre, sin restricciones, ya que en este nivel se elaboran juicios y valoraciones personales en función de la lectura propuesta y a partir de lo que el estudiante haya comprendido del texto.

La prueba de comprensión lectora tuvo un máximo de cuatro preguntas de nivel crítico, para las cuales se redactó tres alternativas. El estudiante tuvo la libertad de seleccionar una opción de acuerdo con la interpretación que le dio a 
las cuatro lecturas propuestas. Ninguna de las alternativas que eligieron se consideró incorrecta.

Esto debido a que el nivel crítico valorativo es subjetivo en relación con los otros niveles de la comprensión lectora. A diferencia de los niveles literal e inferencial, el nivel crítico presenta las opiniones, creencias, es decir diferentes interpretaciones acerca de un tema propuesto. Por tal motivo, no se puede circunscribir al estudiante a una sola alter nativa como respuesta en una prueba de comprensión lectora.

\section{Conclusiones}

Los resultados señalan que, a mayor comprensión lectora, mayor redacción académica, y a menor comprensión lectora, menor redacción académica. Existe una correlación débil, pero a la vez significativa, esto depende de las experiencias que el estudiante tenga en relación con la lectura, y de la información que maneje acerca de diferentes tópicos, ya que, de esta manera, podrá plasmarlo en un texto académico.

En cuanto a la relación entre el nivel literal y la redacción académica, los resultados indican que, a mayor nivel de comprensión lectora, mayor redacción académica, y a menor nivel comprensión lectora, menor redacción académica. Existe una correlación débil, pero a la vez significativa, lo cual se logra cuando el estudiante es capaz de realizar una buena comprensión lectora en el nivel literal, entonces podrá seleccionar y plasmar el tema principal del texto, así como las ideas principales y secundarias.

En cuanto a la relación entre el nivel inferencial y la redacción académica, los resultados determinan que, a mayor nivel de comprensión lectora, mayor redacción académica, y a menor nivel de comprensión lectora, menor redacción académica. Existe una correlación débil, pero a la vez significativa, es decir que, si el estudiante es capaz de elaborar inferencias, además de demostrar su conocimiento acerca del mundo, podrá seleccionar y modificar la información que deberá redactar en el texto académico.

Acerca de la relación entre el nivel crítico y la redacción académica, sin embargo, los resultados demuestran que no existe relación entre el nivel crítico de la comprensión lectora y la redacción académica. La razón este resultado es que la prueba de comprensión lectora presentó alternativas que, en ningún caso, fueron incorrectas, ya que el nivel crítico valorativo es subjetivo. El estudiante eligió una opción de acuerdo con la interpretación que le dio al texto. 


\section{Roxana Abanto Atauje}

\section{Referencias bibliográficas}

Arrieta, B., Batista, J., Meza, R., \& Meza, D. (2006). La comprensión lectora y la redacción académica como centro del currículum. En Laurus, 12(21), 1-10. Venezuela: Universidad Pedagógica Experimental Libertador. Recuperado de https://www.redalyc.org/pdf/761/76102107.pdf

Boudghene, D., \& Bettadj, N. (2014). Papel del profesor para fomentar la competencia lectora y escrita en la clase (Tesis de Licenciatura). Universidad Abou Bakr Belkaid-Tlemcen, Argelia. Recuperado de https://bit.ly/2lr8rkz

Bono, A. \& Barrera, S. de la. (1997). Los estudiantes universitarios como productores de textos. Una experiencia de docencia compartida. En Lectura y Vida, 1-10. Recuperado de https://bit.ly/2roelzu

Cáceres, A., Donoso, P., \& Guzmán, J. (2012). Comprensión lectora. «Significados que le atribuyen las/los docentes al proceso de comprensión lectora en NB2» (Tesis de Licenciatura). Universidad de Chile, Santiago.

Capomagi, D. (2013). La escritura académica en el aula universitaria. Revista de Educación y Desarrollo, 25, 29-40. Recuperado de http://bit.ly/33a8hqn

Congreso de la República de Perú. (2014). Nueva Ley Universitaria, Ley n. ${ }^{\circ} 29904$. El Peruano, 31(12914), 1-21. Recuperado de http://bit.ly/32y8vns

Cassany, D., Luna, M., \& Sánz, G. (2003). Enseñar lengua. Barcelona: Graó. Recuperado de https://bit.ly/2ephmba

Díaz-Barriga, F, \& Hernández, G. (2002). Estrategias para el aprendizaje significativo II: comprensión y composición de textos. En Autores (Eds.), Estrategias docentes para un aprendizaje significativo. Una interpretación constructivista ( $\mathrm{p}$. 310). Recuperado de https://bit.ly/2t4wer2

Espinosa, C. (1998). Lectura y escritura. Buenos Aires: Novedades Educativas.

Gordillo, A., \& Flórez, M. (2009). Los niveles de comprensión lectora: hacia una enunciación investigativa y reflexiva para mejorar la comprensión lectora en estudiantes universitarios. En Revista Actualidades Pedagógicas, 53, 1-13. Recuperado de https://bit.ly/2qiibsp

Natale, L., \& Stagnaro, D. (2012). Desarrollo de habilidades de lectura y escritura en la trayectoria académica del ingeniero: la experiencia de un programa desafi ante e innovador. Revista Argentina de Enseñanza de La Ingeniería, 13(23), 45-52. Recuperado de http://bit.ly/2x1bjwt

Ocde. (2016). Resultados Clave Pisa 2015. Organización Para La Cooperación y El Desarrollo Económicos, 1-15. Recuperado de http://bit.ly/2cqmvid 
Comprensión lectora y redacción académica en estudiantes...

Ochoa, L. (2008). Comunicación oral argumentativa. Estrategias didácticas. Borgotá: Cooperativa Editorial Magisterio. Recuperado de https://bit.ly/2gseg2r Olave-Arias, G., Rojas-García, I., \& Cisneros-Estupiñán, M. (2013). Deserción universitaria y alfabetización académica. Educación y Educadores, 16(3), 455-471.

Pérez, H. (2006). Comprensión y producción de textos. Bogotá: Cooperativa Editorial Magisterio. Recuperado de https://bit.ly/2fob4to

Perilla, A., Rincón, G., Saúl, J., \& Salas, M. (2004). El mejoramiento de los procesos de comprensión de textos académicosen el ámbito universitario. Recuperado de https://bit.ly/2qiibaq

Instituto de Opinión Pública. (2015). Encuesta de Opinión Nacional Urbano Rural «Ciudadanía, hábitos de lectura y prevención de desastres» - 2015. Boletín del Instituto de Opinión Pública, 11(137), 1-13. Recuperado de http://repositorio. pucp.edu.pe/index/handle/123456789/52103

Pineda, M., \& Lemus, F. (2000). Lenguaje y Expresión 1. Lectura y comunicación escrita. Pearson Education. Recuperado de https://bit.ly/2tq32xo

Sánchez, A. (2003). Elementos de escritura académica. En Revista virtual Universidad Católica del Norte, 11. Recuperado de https://bit.ly/1prophv 